\title{
On the significance of form-induced stress in rough wall turbulent boundary layers
}

\author{
Costantino MANES ${ }^{1,2}$, Dubravka POKRAJAC ${ }^{2}$, \\ Omduth $\mathrm{COCEAL}^{3}$, and Ian $\mathrm{McEWAN}^{2}$ \\ ${ }^{1}$ Institute for Snow and Avalanche Research, SLF, Davos Switzerland \\ e-mail: manes@slf.ch (corresponding author) \\ ${ }^{2}$ School of Engineering, University of Aberdeen, \\ Aberdeen, Scotland, United Kingdom; e-mail: d.pokrajac@abdn.ac.uk \\ ${ }^{3}$ Department of Meteorology, University of Reading, Reading, United Kingdom \\ e-mail: o.coceal@reading.uk
}

\begin{abstract}
This paper presents a review of recent experimental and numerical studies which deal with the analysis of form-induced stress in rough wall turbulent boundary layers. The aim of the paper is to assess the importance of this stress for various rough wall geometries and flow conditions. Analysis of the significance of form-induced stress is first performed by comparing its magnitude with the magnitude of Reynolds stress for each data set available in literature. Then, by selecting a special set of data, we analyze the comparison between the gradients of both stresses. We point out that the comparison of stress gradients gives a different perspective on the role of form-induced stress in rough wall boundary layers.
\end{abstract}

Key words: form-induced stress, rough wall boundary layers, Reynolds stress.

\section{INTRODUCTION}

Double Averaged (DA) transport equations have proved to be an efficient tool for investigating transport processes occurring within the roughness layer of rough wall boundary layers. So far, most studies have focused on the

(C) 2008 Institute of Geophysics, Polish Academy of Sciences 
use of the DA momentum equations to interpret data obtained from experiments and numerical simulations. In these studies, the contribution of different terms was assessed in order to highlight the dominant momentum transport mechanisms. In this context significant attention has been paid to quantify the importance of the "Form-Induced" (FI) stress (also called "Disper-sive Stress" by the atmospheric science community). FI stress is a product of spatial averaging, just as the Reynolds stress is a product of time averaging. It represents the stress which arises from the covariance of the spatial fluctuations of the time-averaged flow.

In numerical studies based on DA momentum equations, FI stress is usually neglected, since it is considered much smaller than the Reynolds stress. Behind this assumption there is the hypothesis that most of the momentum within the roughness layer is carried by turbulence. Although this hypothesis is often valid, various studies have shown that FI stress cannot always be neglected and that in certain cases it can have a magnitude comparable with that of the Reynolds stress. In existing literature, the significance of FI stress has been investigated for various roughness geometries and flow conditions and it is the aim of this paper to review the results obtained from recent experiments and numerical simulations. These results are then reinterpreted, where possible, by analyzing momentum balance based on stress gradients rather than on stress values. This type of analysis gives a different perspective on the importance of FI stress.

The paper starts by defining the DA momentum equations used as the basis for reviewing results from the literature. The review of existing data is presented, as is usually done, by assessing the magnitude of FI stress for various rough surfaces and flow conditions. In the discussion section we select a special data set to show how significantly different insight is gained on evaluating the contribution of FI stress to momentum balance, if stress gradients rather than stress magnitudes are compared. In this section we also discuss the difficulties which arise when one wants to parameterize the FI stress term in the momentum balance equation.

\section{DOUBLE AVERAGED MOMENTUM EQUATION}

In this paper we use the equations presented in Nikora et al. (2007), for twodimensional, steady, uniform and turbulent open channel flow conditions over a rough wall. For these flow conditions, the DA momentum equation in the streamwise direction is given as

$$
\rho \varphi g S_{b}=f_{x}-\frac{\partial \varphi \tau_{x z}}{\partial z}
$$

where

$$
\tau_{x z}=\rho\left\langle v \frac{\partial \bar{u}}{\partial z}\right\rangle-\rho\left\langle\overline{u^{\prime} w^{\prime}}\right\rangle-\rho\langle\tilde{u} \tilde{w}\rangle
$$


and $f_{x}$ is the total drag per unit volume. In the above equations the straight over-bar and the angle brackets denote respectively the time and spatial average of flow variables whereas the prime and the wavy over-bar denote respectively the time fluctuations and the spatial disturbances, i.e., $u^{\prime}=u-\bar{u}$ and $\tilde{u}=\langle\bar{u}\rangle-\bar{u} ; x, z$ are the stream wise and bed normal coordinate respectively ( $x$ is positive in the mean direction of the flow whereas $z$ is positive from the wall towards the boundary layer top); $u$ and $w$ are the velocity components along $x$ and $z$, respectively; $\rho$ is the density; $g$ is the gravity acceleration; $S_{b}$ is the bed slope; $v$ is the kinematic viscosity; $\varphi$ is the porosity equal to the ratio between the volume occupied by the fluid and the total averaging volume ( $\varphi=1$ above the roughness top). Volume averaging introduces additional terms with respect to the traditional time-averaged Reynolds equations. These are the FI stress $-\rho\langle\tilde{u} \tilde{w}\rangle$, which becomes a part of the fluid shear stress given by eq. (2), and the total $\operatorname{drag} f_{x}$. The latter represents the sum of the form drag and the viscous drag, i.e., the drag that the fluid exerts on individual roughness elements (per unit height and unit plan area of the flow) due to pressure and viscous forces; $f_{x}$ is non zero only below the roughness elements. The FI stress is a product of spatial averaging just as Reynolds stress is a product of time averaging and represents the momentum flux induced by the heterogeneity of the time-averaged flow.

\section{REVIEW OF EXISTING DATA ON FORM-INDUCED STRESS}

FI stress depends on the details of the time-averaged flow around individual roughness elements, which in turn depends on the roughness geometry and the features of the turbulent flow above the roughness. In this section we present results on FI stress for flows over vertical rods, cubes, spheres, twodimensional artificial dunes and gravel having different spacings and arrangements. All these roughness geometries are not easy to identify by general parameters which take into account, shape, spacing and dimension of each roughness element composing the surface. Furthermore, some of them have well defined shapes (cubes, spheres, artificial dunes and rods) whereas other resemble more a random field of elevations (gravel beds). For these reasons we proceed with the analysis by commenting results case by case without labeling each roughness with a series of parameters describing it. An attempt to identify the roughness characteristics most influencing FI stress behavior is however presented in the section devoted to discussion.

Since the work of Wilson and Shaw (1977), the Double Averaging (hereafter we use DA as an abbreviation for either "Double Averaged" or "Double Averaging") methodology has become a standard for data interpretation in canopy flows. It seems therefore reasonable to start our analysis from this class of rough wall boundary layers. Canopy flows are usually considered to be those turbulent boundary layers occurring over trees or plants. 
In laboratory studies such type of roughness is usually modelled as simple vertical rods which, despite lacking in leaves and branches, still induce flow properties similar to real plant canopies. Many detailed velocity data sets are available in literature within the roughness layer of rod type roughness. The recent experiments of Poggi et al. (2004a) and (2004b) provide an extensive data set which has been used to investigate the dependence of turbulence characteristics on canopy density. Velocities were measured in a water flume with varying canopy density from 67 to $1072 \mathrm{rod} / \mathrm{m}^{2}$ (equivalent to an element area index $a=0.27-4.27$, where $a=n b H_{c p} / S, n$ is equal to the number of roughness elements, $b$ their width, $H_{c p}$ is their height and $S$ is the total area).Velocity measurements were performed at many points above and within the roughness layer and therefore FI stress could be estimated reasonably well throughout the boundary layer depth. It was noted by Poggi et al. (2004a) that FI stress was negative close to the canopy top and positive below, indicating upward and downward "Form-Induced" momentum transport near the canopy top and bottom, respectively. For dense canopy density FI stress was found to be negligible hence substantiating the data of Raupach (1994) and Kaimal and Finnigan (1994). Instead, for sparse roughness FI stress reached up to $30 \%$ of the total stress in the lower part of the canopy $\left(z / H_{c p}<0.5\right.$, where $z=0$ at the canopy bottom). Non negligible FI stress in sparse canopies was also found by Bohm et al. (2000). Above the canopy top, FI stress was found to be negligible for all the roughness densities investigated.

Among the atmospheric science community, the DA technique has been recently applied for the study of boundary layers over urban-like rough surfaces. In a recent paper, Coceal et al. (2006) present results from Direct Numerical Simulations (DNS) performed for turbulent boundary layers over cubes arranged in staggered and aligned configurations. The simulated flow is maintained by a constant pressure gradient and the roughness Reynolds number, (i.e., $R e_{*}=u_{*} H_{c} / v$, where $H_{c}$ is the cubes height and $u_{*}$ is the shear velocity) is within the fully rough regime. The roughness geometries used in this study resemble the structure that buildings and streets form in real cities and hence is used as a reference model for the study of transport mechanisms in urban environments.

Results on the momentum balance for both cube arrangements are shown in Fig. 1. As for canopy flows, FI stress is negligible above the roughness elements, but becomes significant below the roughness tops reaching up to $20 \%$ of the total shear stress $\tau_{0}$, where $\tau_{0}=\tau_{x z}\left(z=H_{c}\right)$, and $z=0$ at the cubes bottom. FI stress for the two roughness configurations shows significant differences in magnitude and also in sign (the sign varies with height for the aligned configuration), indicating a strong dependence of FI stress on roughness arrangement. 


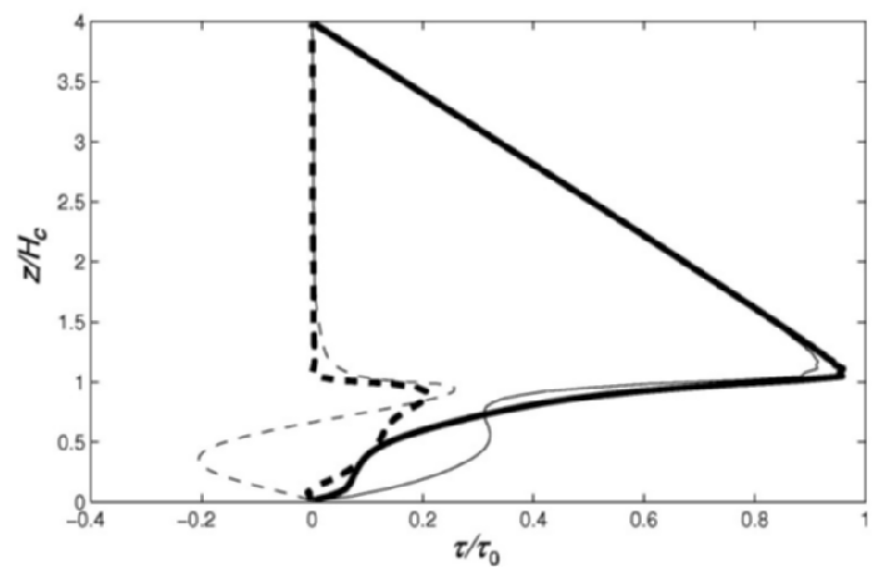

Fig. 1. FI (dashed lines) and Reynolds stress (solid lines) for the staggered (thick lines) and aligned (thin lines) cube arrangement (data from Coceal et al. 2006); $H_{c}$ is the cube height; $z / H_{c}=1$ at the roughness tops; $\tau_{0}$ is the shear stress measured at the roughness tops.

In hydraulics, the DA methodology is receiving considerable attention for the study of open channel flow hydrodynamics (e.g., Nikora et al. 2001, 2007, Maddux et al. 2003, Campbell et al. 2005, McLean and Nikora 2006, Manes et al. 2007). Recently, McLean and Nikora (2006) have applied the DA methodology for interpreting existing data consisting of detailed velocity measurements in open channel flows over two-dimensional artificial dunes and cobble beds. Dune experiments were carried out by varying flow conditions and bed form aspect ratio (details on the experimental set up can be found in McLean et al. 1994). The aspect ratio, i.e., the wavelength $\lambda$ to dune height $H_{d}$ ratios were 20 and 10 . It was observed that, FI stress reached up to 50 and $75 \%$ of the bed shear stress for $\lambda / H_{d}=10$ and 20 , respectively (the bed shear stress was determined from the extrapolation of the Reynolds stress profile to the mean bed level). The contribution of FI stress appeared significant (i.e., comparable to the Reynolds stress) throughout the dune height for $\lambda / H_{d}=20$ whereas it was rapidly decaying with $z$ for $\lambda / H_{d}=10$. Interestingly, FI stress was negative at the dune crests for both roughness spacings, indicating once again an upward momentum transport contribution to the total flux. It was shown that the higher contribution of FI stress for the $\lambda / H_{d}=12$ spacing was due to a significant increase in the spatial variations of the stream-wise velocity components, i.e., $\left\langle\tilde{u}^{2}\right\rangle / u_{*}^{2}$. McLean and Nikora (2006) related this increase to a smaller relative extension of the separated flow region in the case of the longer dunes.

The data on the cobble bed presented by McLean and Nikora (2006) relate only to the flow region above the roughness crests where FI stress was observed to be extremely small. However, recent studies suggest that FI 
stress might be non negligible (i.e., up to $30 \%$ of the bed shear stress) in open channel flows over gravel beds both above (Campbell et al. 2005) and below the roughness tops (Aberle 2006).

As one can see, most of the information on FI stress behavior comes from empirical observations and theoretical arguments are lacking. To the author's knowledge, the only study presenting a theoretical approach is that by Gimenez-Curto and Corniero Lera (1996). In this work, the authors point out that a necessary condition for FI stress to be non-zero is the presence of vorticity in the time-averaged flow. Furthermore, their theoretical considerations lead to the conclusion that the contribution of FI stress to the total shear stress may increase with decreasing relative submergence of the flow, i.e., the ratio between flow depth and roughness height. This theoretical finding is somewhat substantiated by the experimental data of Manes et al. (2007). In this study, experiments were carried out in open channel flows over 1 layer of spheres packed in a cubic pattern. Streamwise and vertical velocities were measured by means of Particle Image Velocimetry (PIV) in two central cross sections of the flume (Fig. 2), one over the tops of the spheres (top section) and another over the points at which they touch (Valley section). This was done in order to capture adequately the spatial heterogeneity of the flow within the roughness layer. Two flow conditions were investigated with two different values of relative submergence, namely $D / H_{s}=1.8$ and 3.5, where $D$ is the flow depth measured from the spheres tops to the free surface and $H_{s}$ is the spheres height. For the lower submergence case, the relative magnitude of FI stress (i.e., $\tau_{f} / \tau_{0}=-\langle\tilde{u} \tilde{w}\rangle / u_{*}^{2}$, where $\tau_{0}$ is the shear stress measured at the spheres top) is almost twice as big as for the higher submergence case (Fig. 3a). However, this increase of FI stress is not accompanied by a significant relative increase of spatial variations of the flow (Fig. $3 \mathrm{~b}$ and 3c), contrary to what is predicted by Gimenez-Curto and Corniero Lera (1996). Manes et al. (2007) relate this increase rather to the development of different flow patterns around the roughness elements which induce higher FI stress for lower $D / H_{s}$. It should be noted that the data on 2-D dunes presented by McLean and Nikora (2006) partly substantiate this argument. In fact, for the $\lambda / H_{d}=20$ case FI stress increases significantly below the dunes crest (i.e., for $z / H_{d} \leq 0.3$ ) by lowering $D / H_{d}$ from 13.65 to 3.95 . Furthermore, as in

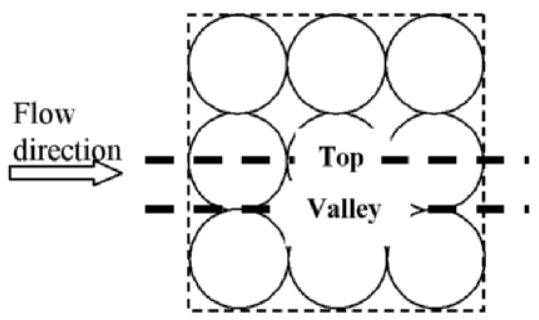

Fig. 2. Location of the measurement sections in Manes et al. (2007). 

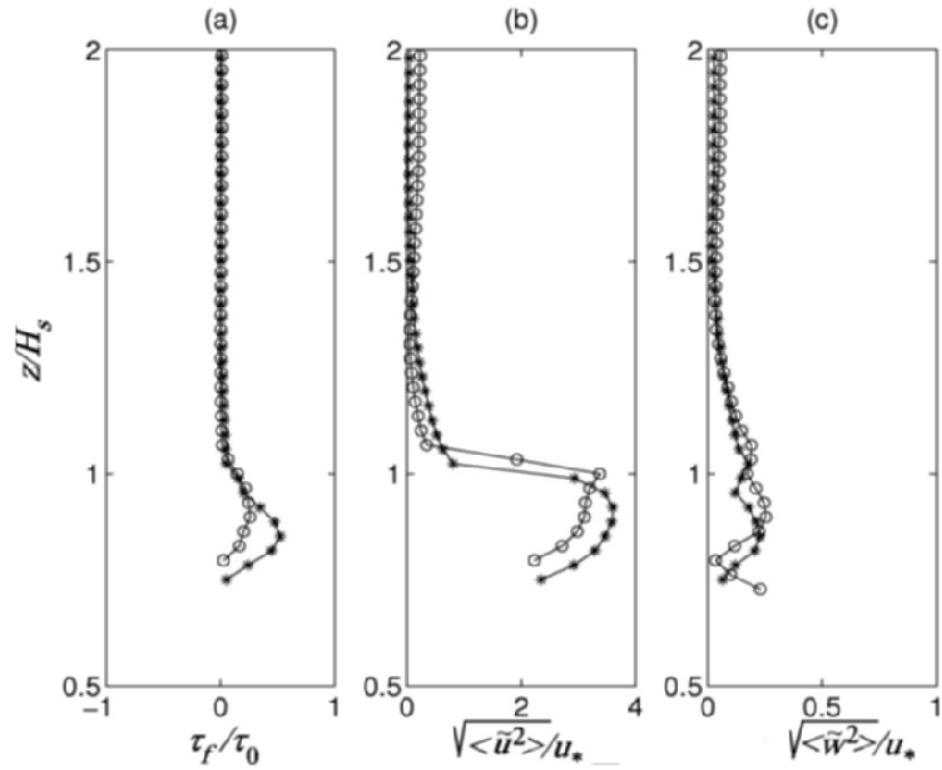

Fig. 3. Dependence of form-induced stress on relative submergence. Lines with circles stand for experiment with $D / H_{s}=3.5$, whereas lines with asterisks for the experiments having $D / H_{s}=1.8$ (data from Manes et al. 2007); $\tau_{0}$ is the shear stress measured at the roughness tops and $u_{*}=\sqrt{\tau_{0} / \rho} ; z / H_{s}=1$ at the sphere tops.

Manes et al. (2007), this increase is not accompanied with an increase in spatial variations of the flow, i.e., $\sqrt{\left\langle\tilde{u}^{2}\right\rangle} / u_{*}$ and $\sqrt{\left\langle\tilde{w}^{2}\right\rangle} / u_{*}$. In contrast to this, for the steeper dunes, i.e., $\lambda / H_{d}=10$, the relative magnitude of FI stress does not vary with flow conditions. This suggests that the dependence of FI stress on flow conditions should be verified by more experimental or numerical tests since the available data and theoretical considerations lead to contradictory results. Indeed, FI stress is generally difficult to estimate and noisy data might give a wrong perspective. Furthermore, it is not clear, from the physical point of view, why the relative submergence should be the nondimensional parameter most influencing the relative magnitude of FI stress.

It is rather more intuitive to infer just that the relative magnitude of FI stress, i.e., $\tau_{f} / \tau_{0}=-\langle\tilde{u} \tilde{w}\rangle / u_{*}^{2}$ varies with flow conditions if these can significantly influence the time-averaged flow patterns developing around the roughness elements. Due to the complicated nature of the flow within the roughness layer it is difficult to predict how this can happen and what are the nondimensional flow parameters controlling such process. To shed some light into this issue, we make use of the spatial quadrant analysis technique developed by Pokrajac et al. (2007), to analyze the data from Manes et al. (2007) and explore possible causes responsible for variations in the timeaveraged flow structures associated with changes in flow conditions. 
We restrict our analysis to the measurements taken at the height were the maximum in the FI stress profile occurs for the two flow conditions, i.e., $z / H_{s} \sim 0.87$ and (Fig. 3). Quadrant analysis for $\tilde{u} / u_{*}$ and $\tilde{w} / u_{*}$ show that spatial perturbations pertaining to top and valley sections reside in two well separated groups (Fig. 4).

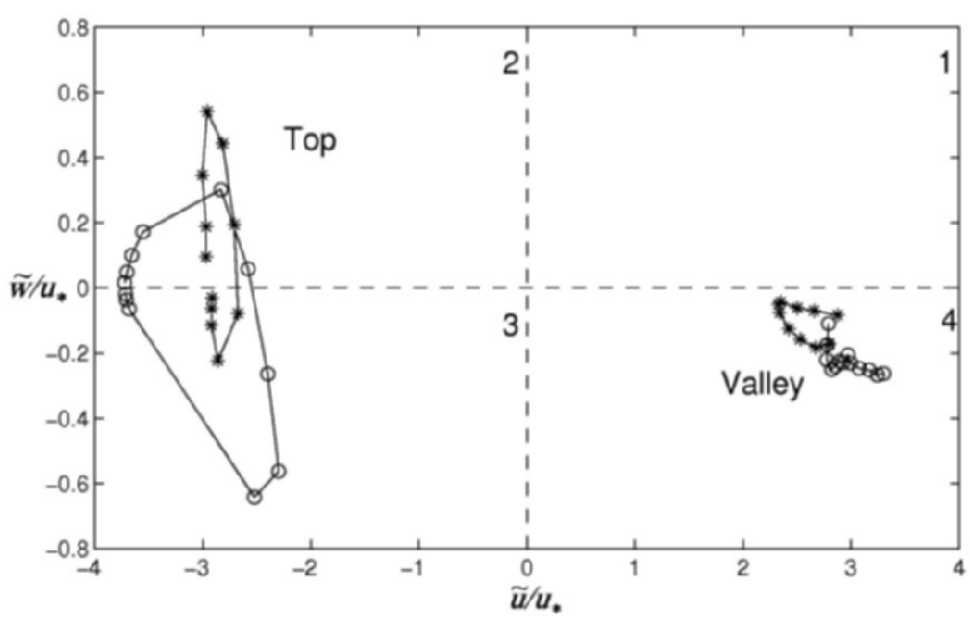

Fig. 4. Quadrant analysis at $z / H_{s} \approx 0.87$ for the experiments of Manes et al. (2007). Symbols are like in Fig. 3.

Valley points are all placed in quadrant 4 and they all contribute to a negative spatial correlation and therefore to a positive FI stress. In contrast, top points are spread in quadrant 2 and 3 which have, respectively, a negative and positive contribution to spatial correlation (i.e., a positive and negative contribution to FI stress). For the flow conditions with higher submergence the contribution of quadrant 3 is larger than in the case with low submergence, and that weakens the overall magnitude of FI stress.

The positioning of top points in the quadrant plot is associated to a waketype mean flow pattern occurring in the top section (Fig. 5). Within the wake region, the flow forms a stationary vortex for which, in the low submergence case, upward vertical velocities are larger than the downward components. In contrast, downward velocities are larger in the high submergence case. Therefore, the uneven spread of points into quadrant 2 and 3 for the top sections is due to development of an "asymmetric" vortex downstream the sphere tops. The different asymmetry influences the positive or negative contribution to FI stress and therefore its overall magnitude. In the case of a perfectly symmetric wake, the contribution to the total FI stress from top points would be zero.

A definitive explanation to justify the development of different vortices for different flow conditions is difficult to provide here. However, we argue 


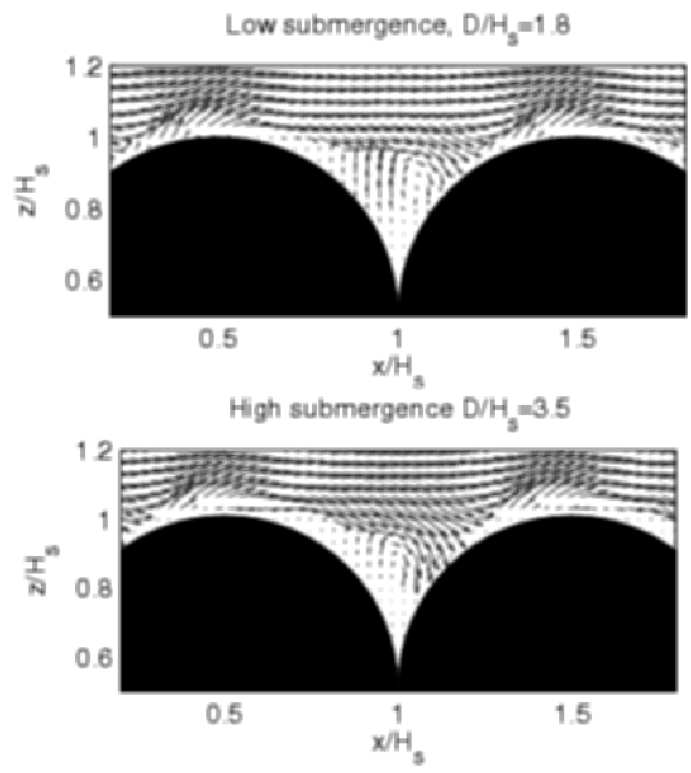

Fig. 5. Time-averaged flow pattern in the top section for the sphere experiments of Manes et al. (2007).

that this could be the result of a shift in the separation point around the spheres. The following argument motivates this hypothesis.

It has been shown by Poggi et al. (2004b) that the drag coefficient $C_{d}$ of rods ion canopy flows, monotonically decreases with increasing local element Reynolds number $R e=\bar{u} d / v$, where $\bar{u}$ is the local time averaged velocity measured upstream from a rod of diameter $d$. This is in contrast to the classical behavior of an isolated cylinder for which $C_{d}$ reaches a plateau for high $R e$ (it is generally argued that such a phenomenon is caused by the sheltering of consecutive roughness elements, see Raupach and Thom 1981). We argue that a variation of $C_{d}$ with $R e$ may be related to a shift in the separation point around the singular roughness element. Let us assume that this argument may also apply to other roughness geometries. In the case of the spheres experiments, the increase in relative submergence is associated with an increase of the local Reynolds number at any height within the roughness layer (i.e., mean velocities below the sphere tops were higher for the high relative submergence case). Therefore, it is plausible to assume that a downstream shift in the separation point occurred between the low and the high submergence case. Figure 5 shows that, since the separation point is at the downstream half of the sphere, the downstream shift produces a downwards velocity which weakens the upward motion within the stationary vortex.

We point out that a shift of the separation point may not be the only mechanism influencing the mean flow patterns among the roughness elements. 
Therefore, it may not provide a general argument to explain the dependence of FI stress with flow conditions. Indeed, it is probably not appropriate to justify the variation of FI stress observed for the dune experiments of McLean and Nikora (2006). This is because a dependence of separation point with element Reynolds number is expected to be more significant for smooth roughness geometries, such as spheres, than for more irregular and sharp edged shapes, such as cubes or artificial dunes. This is substantiated by the data of Taylor (1988) who found that Reynolds number dependence of drag coefficients for various roughness geometries was stronger for smooth shaped roughness elements than for sharp edged shapes. Therefore, more detailed experiments or numerical simulations are needed to reveal how the structure of the time-averaged flow can change by varying bulk flow conditions.

\section{DISCUSSION}

The data presented in the previous section allow us to draw some conclusions on the behavior of FI stress:

a FI stress is often negligible above the roughness tops whereas it increases significantly below this level.

- Within the roughness layer of all the rough surfaces investigated, FI stress becomes significant but reaches at the very most the same magnitude as the Reynolds stress, being smaller most of the times.

a FI stress is more significant when the roughness is more sparse. This is evident from the canopy flow experiments of Poggi et al. (2004a) and the dunes experiment of McLean and Nikora (2006).

a FI stress scaled with the surface shear stress may depend on flow conditions (Manes et al. 2007, McLean and Nikora 2006, and Fig. 3a).

Among the conclusions listed above, the first and the second point seem to suggest that the FI stress is less important than Reynolds stress and that turbulence is generally the strongest driving force for vertical momentum transfer through rough wall boundary layers. However, one has to be careful since it is the gradient of the stress that enters the DA momentum equation (1), which governs the mean dynamics within the roughness layer. Therefore, information on the roughness layer flow dynamics should be obtained from the analysis of the stress gradient terms rather than the stress components. The same argument has been recently used by Wei et al. (2005) and Klevicki et al. (2007) who analyze the structure of smooth wall turbulent boundary layers by assessing the gradients of viscous and Reynolds stress. We now want to perform a similar analysis for the Reynolds and FI stress gradients. Estimating values of stress gradients requires very detailed velocity data which can only be provided by numerical simulations. In this review, 
we take advantage of the DNS of Coceal et al. (2006) for flows over cubes (these data are also presented in Coceal et al. 2008 in this special issue). Figure $6 \mathrm{a}$ and $6 \mathrm{~b}$ show the ratios between FI and Reynolds stress and the ratio between their gradient. According to Pokrajac et al. (2008) care should be taken when assessing stress gradients at the roughness tops since at this level the stress might not be a continuous function of $z$. This implies that at this level $\tau(z)$ may not be differentiable and therefore its gradient may not be defined. In Figs. 6 and 7 we show the values of the stress gradients calculated at the roughness tops for completeness, however, we acknowledge that due to the aforementioned problem their values might just be a result of the discrete nature of the DNS data.

Figure 6 shows that although the ratio between the stress magnitudes is always less than or equal to one, the ratio between the gradients of FI and Reynolds stress is much bigger for both roughness configurations. Indeed, for the aligned configuration it goes up to 20, maintaining large values for a significant part of the roughness height, i.e., for $0.52<z / H_{c}<0.72$ and $0.45<z / H_{c}<0.5$ (the discontinuity of the gradient ratio for the aligned profile is caused by the fact that Reynolds stress becomes zero at $z / H_{c}=0.5$ ). For the staggered configuration the stress gradient ratio goes up to 5 and also keeps a value larger than 1 for a large part of the roughness height, i.e., $0.05<z / H_{c}<0.35$.

(a)

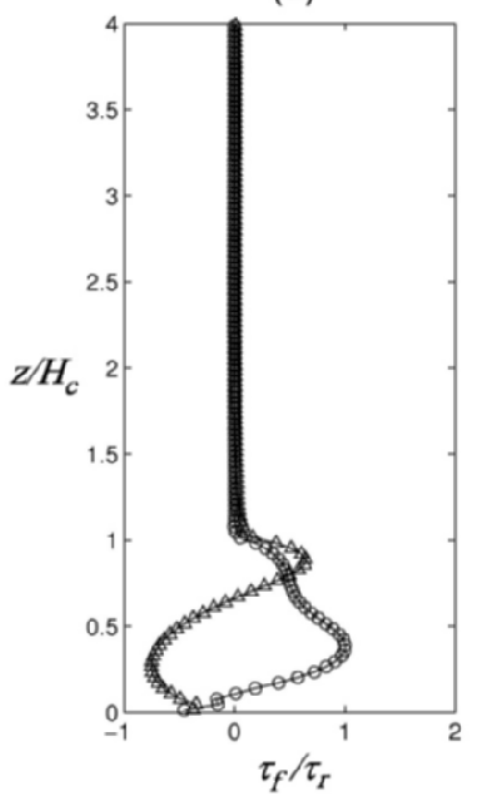

(b)

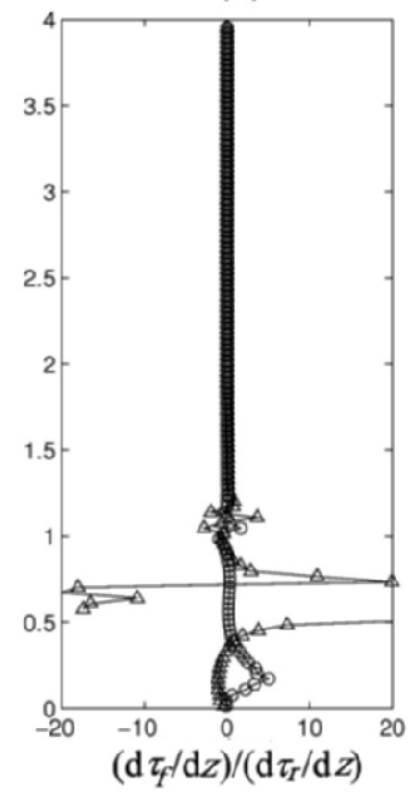

Fig. 6: (a) Ratios of FI and Reynolds stresses; (b) Ratios between FI and Reynolds stress gradients. Circles and triangles relate to the staggered and aligned cube configuration, respectively. 
Clearly, the conclusions that one can draw from the standard stress and stress gradient analysis differ significantly. From the stress analysis one would say that FI and Reynolds stress are at the very most just comparable. Instead, from the stress gradient analysis one can definitely say that there are flow regions where FI fluxes represent the dominant mechanism. This means that, despite being smaller than the Reynolds stress, FI stress can have much steeper gradients and hence contributes more to the force exerted on the fluid at each elevation. Therefore, in order to gain insight into the dynamics of the roughness layer, this analysis shows that it might be misleading to evaluate the significance of FI stress just from its magnitude.

In order to have a clear picture of the mean dynamics of the flow, we present the total force balance for both roughness configurations in Fig. 7. This includes the gradient of FI and Reynolds stress and also the total drag force per unit area. The data are presented normalized with the total pressure gradient (pressure gradient is analogous to gravity in eq. 1). Above the roughness tops, the force balance is between the Reynolds stress and pressure gradient, in fact their ratio is equal to one. Below the roughness tops Fig. 7 further substantiates the arguments presented above. In particular that there are flow regions where the Reynolds stress gradients are almost zero and where therefore, it is conceivable to assume that the flow is maintained by a balance between the pressure gradient and the sum of drag and FI stress gradients.
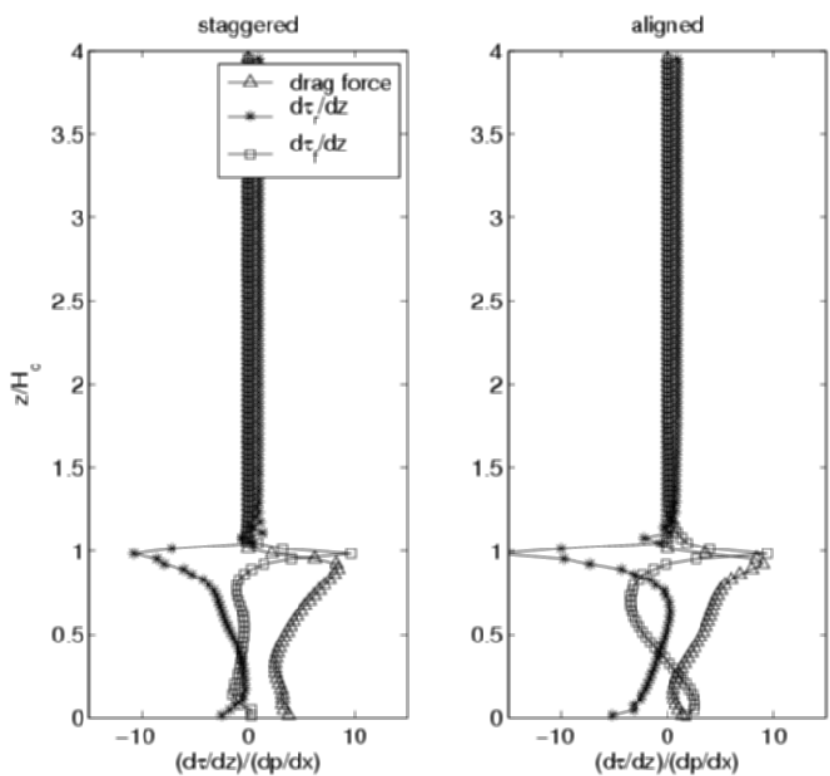

Fig. 7. Momentum balance for staggered and aligned cube configurations; $\tau_{f}$ stands for FI stress and $\tau_{r}$ for Reynolds stress. Data are normalized with the streamwise pressure gradient. 
So far the effects of FI stress in numerical models have always been neglected and only the Reynolds stresses were considered. The parameterization of the Reynolds stress is usually based on eddy viscosity theories which however do not work well within the roughness layer. In this flow region, they suffer the problem of non-local momentum exchange and the absence of a unique length scale influencing the turbulent motion. Eddy diffusivity approaches can be successfully applied only where turbulence is dominated by one length scale, e.g., in the log layer of turbulent boundary layers where the only length scale of turbulence is the distance from the wall. This implies that there is a large degree of tuning and uncertainty when matching turbulence properties of the flow, eddy diffusivity and Reynolds stress within the roughness layer.

We argue that, applying an eddy diffusivity technique for the parameterization of FI stress (as suggested by Gimenez-Curto and Corniero Lera 1996) is often impossible and where possible, not convenient. In fact, we have seen that FI momentum fluxes are often negative, reflecting an upward contribution to the momentum flux. However, the DA mean velocity gradients are usually positive within the roughness layer, therefore one should include a rather non-intuitive negative FI eddy diffusivity to account for such an effect. Even in the case when counter gradient FI momentum fluxes are absent, dividing the effects of the two shear stress components would not be very beneficial. In fact, due to the pathology of eddy diffusivity techniques in the roughness layer, this would just add more uncertainty because it would imply including more tuning coefficients in the momentum equations which cannot be explicitly and easily linked with flow properties.

We suggest that a separate parameterization of the two terms should be done only in case the perturbed time-averaged flow is dominated by a unique
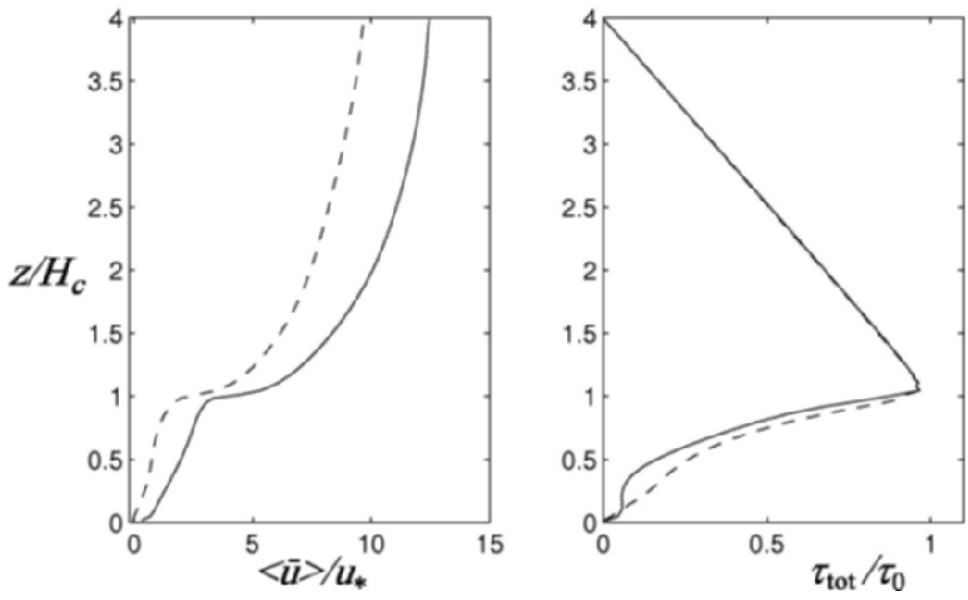

Fig. 8. DA velocity profiles and total fluid shear stress for staggered (dashed lines) and aligned (solid lines) cubes configuration. 
length scale (imposed by the rough surface), which can be consistently linked with a "FI eddy viscosity" of the DA flow. Unless this happens it is definitely more desirable to consider the sum of the effects of the Reynolds and FI stresses in just one total shear stress term. In fact when considering the sum of the stresses at least one does not encounter the problem of counter gradient fluxes because the total fluid shear stress is always positive and hence consistent with a positive gradient of DA velocities (Fig. 8).

From the above analysis, it is clear that the modelling of FI fluxes remains an unsolved problem which probably does not have an easy solution. Predicting FI stress by using standard parameterization implies having a theoretical tool able to describe flows among obstacles which is extremely difficult to do. Only detailed turbulence models, such as Large Eddy Simulation (LES) and DNS are capable of dealing with such tasks. However, the computational cost to compute environmental flows with such models still far exceeds the capability of available computers and a more reasonable alternative has to be found.

\section{CONCLUSIONS}

DA momentum equations have been recently applied to interpret experimental and numerical data in order to investigate momentum transport mechanisms occurring within the roughness layer of rough wall turbulent boundary layers. DA momentum equations are obtained by averaging the standard Navier-Stokes equations first in time and then in space. Spatial averaging gives rise to a stress term produced by the spatial covariance of the timeaveraged velocities, i.e., the FI stress term. In the literature, the significance of FI stress has been investigated for various rough wall geometries and this paper reviews the results obtained from recent experiments and numerical simulations. The main outcomes from this review can be summarized in three points:

- FI stress is often negligible above the roughness elements whereas it can increase significantly below this level. In all of the rough surfaces investigated, FI stress reached at the very most the same magnitude as the Reynolds stress being smaller most of the times. This analysis might lead to the conclusion that turbulence is generally the strongest driving force for momentum transport in rough wall turbulent boundary layers. However, we point out that, it is the gradient of stress which enters the DA equations which governs the mean flow dynamics in a turbulent boundary layer. Therefore, information on the dominant momentum transport mechanisms should be obtained by analyzing stress gradients rather than stress components. By selecting a special data set obtained from DNS of turbulent boundary layers over cubes, we show how in flow regions below the roughness tops, the vertical gradient of FI stress can be much larger than the gradient of 
Reynolds stress (up to 20 times larger). This clearly gives a different perspective on the role of FI stress and shows how an analysis based on stress components could be misleading for assessing the dominant momentum transfer mechanisms. This also suggests that further experimental studies on FI stress should involve flow measurements with a very high spatial resolution in order to be able to properly estimate stress gradients. In this context the use of PIV may be helpfull.

a The magnitude of FI stress clearly depends on roughness arrangement. Recent experiments on open channel flows over rods and artificial dunes indicates that the magnitude of FI stress increases significantly with increasing roughness spacing.

- The relative magnitude of FI stress might depend on flow conditions. Once again we stress that this hypothesis should be confirmed by more experiments or numerical simulations since available data lead to contradictory results. Indeed, dependence on flow conditions does not occur consistently for all roughness geometries investigated. Despite these inconsistencies, we attempt to identify a potential cause which can justify such dependence. We suggest that a change in the position of the separation point around roughness elements could be responsible for a significant variation of the timeaveraged flow pattern, which in turn causes the change in the relative magnitude of FI stress.

\section{References}

Aberle, J. (2006), Spatially averaged near-bed flow field over rough armor layers. In: A.H. Cardoso and R. Ferreira (eds.), Proc. Intern. Conf. on Fluvial Hydraulics, River Flow 2006, Balkema, Brookfield, VT.

Bohm, M., J.J Finnigan, and M.R. Raupach (2000), Dispersive fluxes and canopy flows: Just how important are they? 24th Conf. on Agricultural and Forest Meteorology, 14-18 August 2000, University of California, Davis, CA, 106107.

Campbell, L.J., I.K. McEwan, V.I. Nikora, D. Pokrajac, M. Gallagher, and C. Manes (2005), Bed load effects on hydrodynamics of rough bed open channel flows, J. Hydraul. Eng. ASCE 131, 7.

Coceal, O., T.G. Thomas, I.P. Castro, and S.E. Belcher (2006), Mean flow and turbulence statistics over groups of urban-like cubical obstacles, Bound.-Layer Meteor. 121, 491-519.

Coceal, O., T.G. Thomas, and S.E. Belcher (2008), Spatially-averaged flow statistics within a canopy of large bluff bodies: Results from direct numerical simulations, Acta Geophys. 56, 3. 
Gimenez-Curto, L.A., and M.A. Corniero Lera (1996), Oscillating turbulent flow over very rough surfaces, J. Geophys. Res. 101, C9, 20,745-20,758.

Kaimal, J.C., and J.J. Finnigan (1994), Atmospheric Boundary Layer Flows: Their Structure and Measurement, Oxford University Press, Oxford, 289 pp.

Klevicki, J., P. Fife, T. Wei, and P. McMurtry (2007), A physical model of the turbulent boundary layer consonant with mean momentum balance structure, Phil. Trans. Roy. Soc. Lond. A 365, 823-839, DOI: 10.1098/rsta.2006.1944.

Maddux, T.B., S.R. McLean, and J.M. Nelson (2003), Turbulent flow over threedimensional dunes: 2. Fluid and bed stresses, J. Geophys. Res. 108, F1, 6010, DOI: 10.1029/2003JF000018.

Manes, C., D. Pokrajac, and I.K. McEwan (2007), Double averaged open channel flows with small relative submergence, J. Hydraul. Eng. ASCE 133, 896-904.

McLean, S.R. and V.I. Nikora (2006), Characteristics of turbulent unidirectional flow over rough beds: Double-averaging perspective with particular focus on sand dunes and gravel beds, Water Resour. Res. 42, W10409, DOI: $10.1029 / 2005$ WR004708.

McLean, S.R., J.M. Nelson and S.R. Wolfe (1994), Turbulence structure over two dimensional bed forms: Implications for sediment transport, J. Geophys. Res. 99, C6, 12,729-12,747.

Nikora, V., D.G. Goring, I.K. McEwan, and G. Griffiths (2001), Spatially averaged open-channel flow over a rough bed, J. Hydraul. Eng. ASCE 127, 123-133.

Nikora, V., I.K. McEwan, S.R. McLean, S. Coleman, D. Pokrajac, and R. Walters (2007), Double-averaging concept for rough-bed open-channel and overland flows: Theoretical background, J. Hydraul. Eng. 133, 8, 873-883.

Pokrajac, D., and C. Manes (2008), Interface between turbulent flows above and within rough porous walls, Acta Geophys. 56, 3.

Pokrajac, D., L.J. Campbell, V.I. Nikora, C. Manes, and I.K. McEwan (2007), Quadrant analysis of persistent spatial velocity perturbations over squarebar roughness, Experiments in Fluids 42, 3, 413-423.

Poggi, D., G.G. Katul, and J.D. Albertson (2004a), A note on the contribution of dispersive fluxes to momentum transfer within canopies, Bound.-Layer Meteor. 111, 615-621.

Poggi, D., A. Porporato, L. Ridolfi, J.D. Albertson, and G.G. Katul (2004b), The effect of vegetation density on canopy sub-layer turbulence, Bound-Layer Meteor. 111, 565-587.

Raupach, M.R. (1994), Simplified expressions for vegetation roughness length and zero-plane displacement as functions of canopy height and area index, Bound.-Layer Meteor. 71, 211-216.

Raupach, M.R., and A.S. Thom (1981), Turbulence in and above plant canopies, Ann. Rev. Fluid Mech. 13, 97-129. 
Taylor, P.A. (1988), Turbulent wakes in the atmospheric boundary layer. In: W.L. Steffen and O.T. Denmead (eds.), Flow and Transport in the Natural Environment: Advances and Applications, Springer-Verlag, New York, 270-292.

Wei, T., P. Fife, J. Klevicki, and P. McMurtry (2005), Properties of the mean momentum balance in turbulent boundary layers, J. Fluid Mech. 522, 303-327.

Wilson, N.R., and R. Shaw (1977), A higher order closure model for canopy flow, J. Appl. Meteor. 16, 1198-1205. 